\title{
Selective Oxidative Cleavage of Oleic Acid on Alumina Supported Metal Catalyst
}

\author{
Chayut INTARASUK ${ }^{1,2}$, Varong PAVARAJARN ${ }^{1 *}$, Supakorn SIRIMITTAGOON ${ }^{2}$, and Ratchanon CHANTANUSON ${ }^{2}$ \\ ${ }^{1}$ Department of Chemical Engineering, Faculty of Engineering, Chulalongkorn University, 254 Phayathai Rd, Wang Mai, Pathum \\ Wan District, Bangkok 10330, Thailand \\ ${ }^{2}$ Green Technology Research Co., Ltd. 16th Floor, AIA Capital Center Building 89 Ratchadaphisek Rd., Dindaeng, Bangkok 10400, \\ Thailand
}

\begin{abstract}
Short-chain fatty acids (SCFAs) and medium-chain fatty acid (MCFAs) are valuable raw materials in wide range of chemical and medical applications. They can be converted to other derivatives by known chemical reactions. Unfortunately, both SCFAs MCFAs are not as abundant in nature as long-chain fatty acid (LCFAs). In this work, the oxidative cleavage of oleic acid, which is one of the most abundant unsaturated LCFAs in nature, was studied. The oxidation was induced by hydrogen peroxide catalyzed by a commercial alumina-supported metal catalyst. The products were analyzed by gas chromatography equipped with mass spectroscopy (GC/MS). The results revealed that MCFAs and SCFAs were detected in a product. In addition, aldehydes were also found. In this work, effects of catalyst loading, oleic acid-to-hydrogen peroxide ratio, reaction time were investigated and reported.
\end{abstract}

\section{Introduction}

Nowadays, fats and oil have become more attractive resource as renewable raw materials in oleochemical industries. Unsaturated fatty acids (UFAs), which are abundantly available in nature as the complement of lipids, can be used as a substrate to produce medium-chain fatty acids (MCFAs) and short-chain fatty acids (SCFAs). Generally, these fatty acids are valuable materials and widely used for different industrial applications, such as being used as an intermediates in the production of food preservatives (Gopinger et al., 2015), herbicides, medicines (Ihre et al., 1996), food production, fragrant (Armstrong and Yamazaki, 1986), antibiotic compounds (Huang et al., 2011), biofuels (Yanowitz et al., 2011), lubricants, and plasticizers (Noureddini and Rempe, 1996) (Turnwald et al., 1998). Moreover, short-chain fatty acids can also be converted to other derivatives by chemical reaction to produce biofuels that are environmentally friendly. It is considered that short-chain fatty acids are one of the important building blocks in oleochemical industry.

Generally, SCFAs and MCFAs are obtained from separation of vegetable oils or animal fats. Unfortunately, both SCFAs MCFAs are not as abundant in nature as LCFAs, hence, they are difficult to be separated from the oil. Although SCFAs and MCFAs could be synthesized from petroleum products and natural gas (Spargo, 2003), the process is not only complex and costly, but also contains contaminated residue that makes it unsuitable for consumption.
This research proposes a method to produce SCFAs and MCFAs from LCFAs by oxidation cleavage reaction. In general, the cleavage of carbon-carbon double bond in UFAs following by the oxidation of the cleaved products results in mono- and di- carboxylic acids as shown in Figure 1 (Godard et al., 2016). In industrial scale, oxidative cleavage of UFAs has been developed using ozone as oxidizing agent. Due to hazardous issue, using ozone is not sustainable. Alternative oxidizing agent such as hydrogen peroxide $\left(\mathrm{H}_{2} \mathrm{O}_{2}\right)$ has been studied instead of ozone since it has advantage in not only environmental reasons, but also in economic reasons.

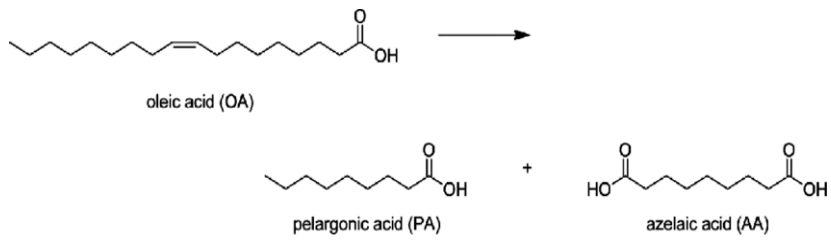

Figure 1. Oxidative cleavage of oleic acid

Transition metals have been used as catalyst for oxidation at mild condition. Several transition metals such as $\mathrm{Os}, \mathrm{Co}, \mathrm{Mo}, \mathrm{Mn}, \mathrm{Fe}, \mathrm{Ru}$, and $\mathrm{W}$ have been investigated for this purpose and have been reported in review paper (Enferadi Kerenkan et al., 2016a). Although homogeneous catalysts have shown excellent

Corresponding author: Varong.P@ chula.ac.th 
performance in oxidation of UFAs (Khlebnikova et al., 2009), they are associated with lack of catalyst recovery. For heterogeneous catalysts, high surface area is needed to enhance contact between the liquid substrate and the solid catalyst (Enferadi Kerenkan et al., 2016a). To achieve such high surface area, mesoporous structure have been employed (Enferadi Kerenkan et al., 2016b). In this work, effects of catalyst loading, oleic acid-tohydrogen peroxide ratio, and reaction time were investigated and reported.

\section{Experimental}

\subsection{Materials}

Oleic acid $\left(\mathrm{C}_{18} \mathrm{H}_{34} \mathrm{O}_{2}, 98 \%\right.$ purity, extrapure grade), hydrogen peroxide $\left(\mathrm{H}_{2} \mathrm{O}_{2}, 30 \% \mathrm{v} / \mathrm{w}\right.$, AR grade), methyl alcohol $\left(\mathrm{CH}_{3} \mathrm{OH}, \mathrm{AR}\right.$ grade $)$, hexane $\left(\mathrm{C}_{6} \mathrm{H}_{14}, \mathrm{AR}\right.$ grade), and hydrochloric acid $(\mathrm{HCl}, 37 \% \mathrm{v} / \mathrm{w}, \mathrm{AR}$ grade) were provided from QReC. Tert-butyl alcohol $\left(\left(\mathrm{CH}_{3}\right)_{3} \mathrm{COH}\right.$, AR grade) was provided from LobaChemie. Sodium sulfate $\left(\mathrm{Na}_{2} \mathrm{SO}_{4}, \quad \mathrm{AR}\right.$ grade $)$ was provided from Fisherchemical. The commercial catalysts supported on alumina ( $1 \%, 10 \%$ Loading) were supplied from Liaoing Haitai Sci-Tech Development Co., Ltd. Standard of methyl oleate $\left(\mathrm{C}_{19} \mathrm{H}_{36} \mathrm{O}_{2}, 1000 \mu \mathrm{g} / \mathrm{ml}\right.$ in hexane $)$, methyl nanoate $\left(\mathrm{C}_{10} \mathrm{H}_{18} \mathrm{O}_{2}, 1000 \mu \mathrm{g} / \mathrm{ml}\right.$ in hexane), dimethyl azelate $\left(\mathrm{C}_{11} \mathrm{H}_{20} \mathrm{O}_{4}, 1000 \mu \mathrm{g} / \mathrm{ml}\right.$ in hexane $)$ were provided from AccuStandard. All chemical reagents were used without further purification.

\subsection{Characterization}

The specific surface area (BET) and pore volume of the catalysts were determined by using the nitrogen adsorption isotherms in volumetric adsorption systems (Micrometrics 3flex surface characterization analyzer).

\subsection{Catalytic test}

Catalytic reactions were carried out in a glass reactor equipped with an oil bath, magnetic stirrer, and reflux condenser. Typically, the reactor was charged with a commercial alumina supported metal catalyst, aqueous $\mathrm{H}_{2} \mathrm{O}_{2}$, oleic acid followed by addition of a tertiary alcohol as solvent. The reaction mixture was heated and kept for a predetermined period of time. After the reaction, the solution was cool down to room temperature. The resultant solution then underwent a derivatization process prior to be analysed by gas chromatography-mass spectrometry (GC-MS).

\subsection{Quantitative Analysis of the Product}

GC-MS was used for separation and quantification of methyl esters of fatty acids. Due to the adsorption problem on the stationary phase in GC columns, the reaction products were esterified to fatty acid methyl ester (FAME) before being analysed. The FAME was prepared according to Ichihara's method (Ichihara and
Fukubayashi, 2010). Firstly, $0.5 \mathrm{ml}$ of reaction product was added into a gas-capped glass tube. Then, $0.3 \mathrm{ml} \mathrm{HCl}$ $(9 \% \mathrm{w} / \mathrm{w}$ solution in methanol) and $1.5 \mathrm{ml}$ of methanol were added. The solution was heated to $80^{\circ} \mathrm{C}$ and kept at this temperature for 2 hours, followed by cooling down to room temperature. The esterified products were extracted by adding $1 \mathrm{ml}$ of hexane and $1 \mathrm{ml}$ of deionized water to separate the organic phase and aqueous phase. Finally, the sample was dehydrated by sodium sulfate. The organic phase was injected into the GC-MS instrument. The GC/MS shimadzu QP2020 was equipped with HPINNOWAX column $(30 \mathrm{~m} \times 0.25 \mathrm{~mm} \times 0.25 \mu \mathrm{m})$. Helium was used as a carrier gas with the flow rate of $30 \mathrm{ml} / \mathrm{min}$. The injector split ratio was fixed at 10:1. The oven temperature program consisted of maintaining at $50^{\circ} \mathrm{C}$ for $1 \mathrm{~min}$, then a ramp rate of $5^{\circ} \mathrm{C} / \mathrm{min}$ to $150^{\circ} \mathrm{C}$ following by a hold-up time of $1 \mathrm{~min}$, and then increased of the rate of $5^{\circ} \mathrm{C} / \mathrm{min}$ to $260^{\circ} \mathrm{C}$ and held for 5 minutes. NIST14 data library was used to analyze data.

\subsection{Calculation}

It should be noted that the data in the results were plotted in relative value, due to term and condition of the collaborated company, which obtained from equation 14.

$$
\begin{gathered}
\% \text { Conversion }=\frac{\text { mole of oleic acid consumed }}{\text { mole of oleic acid before reaction }} \times 100 \\
\% \text { Selectivity }_{A}=\frac{\text { mole of product } A}{\text { mole of oleic acid consumed }} \times 100 \\
\% \text { Yield }_{A}=\frac{\% \text { Conversion } \times \% \text { Selectivity }}{100}
\end{gathered}
$$$$
\text { Relative percentage }=\frac{\% \text { Conversion or } \% \text { Yield }_{A}}{\text { A numeric constant }}
$$

\section{Results and Discussion}

The products obtained from oxidative cleavage of oleic acid in this research are consisted of SCFAs, MCFAs, aldehydes, dicarboxylic acids, and n-oxo carboxylic acids which are shown in Table 1.

\subsection{Effect of catalyst loading}

The effect of catalyst loading on oxidative cleavage of oleic acid using commercial metal catalysts supported on alumina was studied at loading of $1 \%$ and $10 \%$. The results in Figure 2 and Figure 3 show that increasing catalyst loading of $1 \%$ to $10 \%$ resulted in the increase in yield of pelargonic acid (PA) and azelaic acid (AA) regardless of the fact that the overall conversion is 
decreased. This can be anticipated from decreased in surface area of the high-loading catalysts, as shown in Table 2 . The higher yields from $10 \%$ loading catalyst were obtained from the presence of higher content of active metal in the catalyst structure.

Table 1. Summary products from oxidative cleavage of oleic acid

\begin{tabular}{cc}
\hline Functional group & IUPAC name \\
\hline \multirow{3}{*}{ Monocarboxylic acids } & Hexanoic acid \\
& Heptanoic acid \\
& Octanoic acid \\
Nonanoic acid \\
Decanoic acid \\
Aldehydes & Hexanal \\
& Heptanal \\
& Octanal \\
& Nonanal \\
& Decanal \\
Dicarboxylic acids & Octanedioic acid \\
& Nonanedioic acid \\
n-oxo,carboxylic acid & 8-Oxooctanoic acid \\
& 9-Oxononanoic acid \\
\hline
\end{tabular}

\subsection{Effect of hydrogen peroxide-to-oleic acid ratio}

The effect of hydrogen peroxide-to-oleic acid molar ratio were studied at oxidative cleavage of oleic acid using commercial metal catalysts supported on alumina at loading of $1 \%$ and $10 \%$. Results in Figure 2 and Figure 3 show that the increase in hydrogen peroxide-to-oleic acid molar ratio from 1:1 to 10:1 result in increasing oleic acid conversion and increasing yield of PA and AA. It is suggested that the additional amount of hydrogen peroxide shifts the reaction equilibrium to form more products.

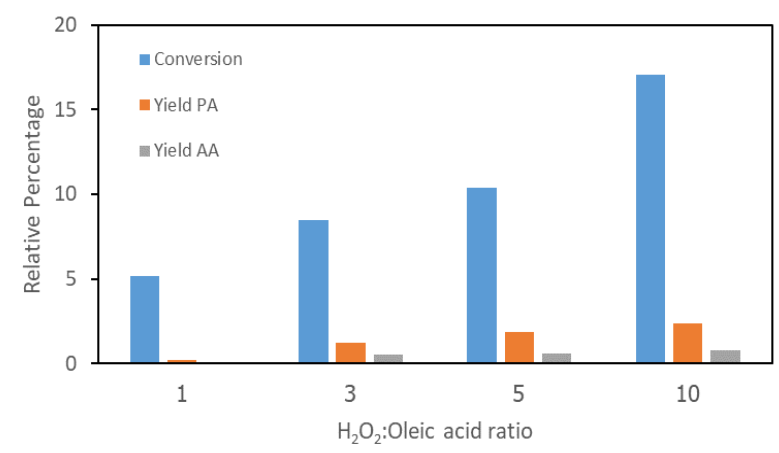

Figure 2. Effect of hydrogen peroxide-to-oleic acid ratio on the oxidative cleavage using $1 \%$ loading catalyst

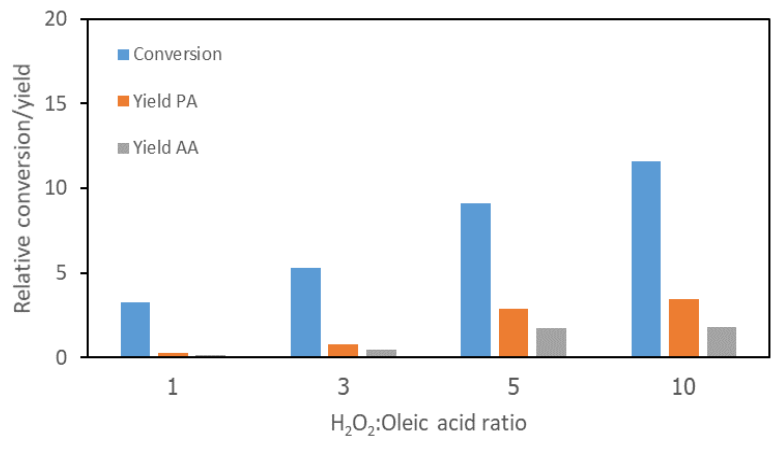

Figure 3. Effect of hydrogen peroxide-to-oleic acid ratio on the oxidative cleavage using $10 \%$ loading catalyst

Table 2. $\mathrm{N}_{2}$-adsorption/desorption isotherms results.

\begin{tabular}{ccc}
\hline \%Catalyst loading & $\begin{array}{c}\mathbf{S}_{\text {BET }} \\
\left(\mathbf{m}^{\mathbf{2}} / \mathbf{g}\right)\end{array}$ & $\begin{array}{c}\text { Pore volume } \\
\left(\mathbf{c m}^{\mathbf{3}} \mathbf{g}\right)\end{array}$ \\
\hline 1 & 290.26 & 0.568 \\
10 & 269.50 & 0.494 \\
\hline
\end{tabular}

\subsection{Effect of reaction time}

The oxidative cleavage of oleic acid using $10 \%$ loading catalyst in a batch reactor was monitored to study the effect of reaction time. The results show that the oleic acid conversion increases significantly within the first 30 minutes. The rate of the reaction slows down after 90 minutes. The yields of PA and AA increase progressively at the start until the reaction time of 120 minutes, after which they declined. This could be anticipated from the decomposed of $\mathrm{H}_{2} \mathrm{O}_{2}$ since the yields of aldehyde products dramatically increase at prolonged reaction time (see Figure 5).

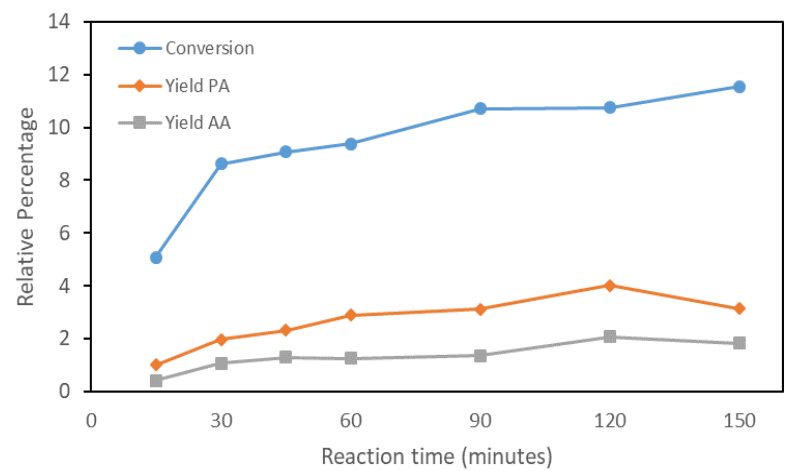

Figure 4. Effect of reaction time on the oxidative cleavage using $10 \%$ loading catalyst (Experimental condition: hydrogen peroxide-to-oleic acid ratio of 10/1) 


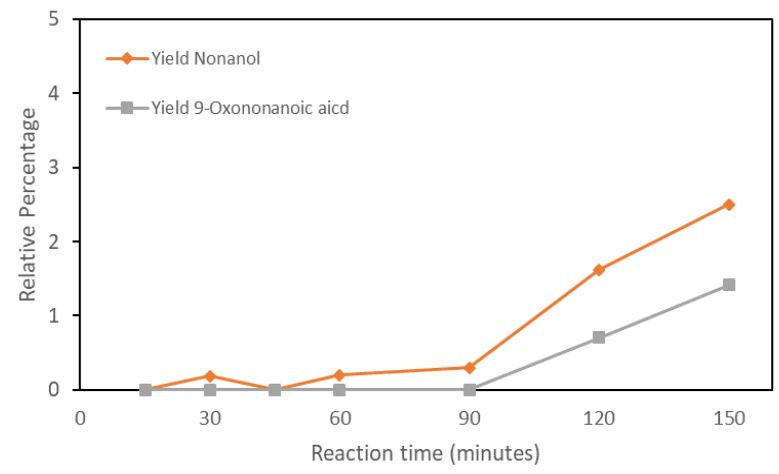

Figure 5. Increasing of aldehyde products with reaction time (Experimental condition: hydrogen peroxide-to-oleic acid ratio of $10 / 1)$

\section{Conclusions}

SCFAs, MCFAs, dicarboxylic acid, and aldehyde products were found as products from oxidative cleavage of oleic acid using commercial catalysts supported on alumina. Oxidative cleavage of oleic acid on $10 \%$ loading catalyst with $\mathrm{H}_{2} \mathrm{O}_{2}$-to-oleic acid molar ratio of 10:1, resulted the highest $\mathrm{PA}$ and $\mathrm{AA}$ yield. It might be concluded that higher catalyst loading, and hydrogen peroxide-to-oleic acid molar ratio could improve acid products yield for the reaction between oleic acid and hydrogen peroxide.

\section{References}

Gopinger, E., V. Ziegler, A.A.d.S. Catalan, E.L. Krabbe, M.C. Elias, and E.G. Xavier; "Whole rice bran stabilization using a short chain organic acid mixture", Journal of Stored Products Research, 61, 108-113. (2015) Ihre, H., A. Hult, and E. Söderlind; "Synthesis, Characterization, and 1H NMR Self-Diffusion Studies of Dendritic Aliphatic Polyesters Based on 2,2Bis(hydroxymethyl)propionic Acid and 1,1,1Tris(hydroxyphenyl)ethane", Journal of the American Chemical Society, 118, 6388-6395 (1996).

Armstrong, D.W. and H. Yamazaki; "Natural flavours production: a biotechnological approach", Trends in Biotechnology, 4, 264-268 (1986).

Huang, C.B., Y. Alimova, T.M. Myers, and J.L. Ebersole; "Short- and medium-chain fatty acids exhibit antimicrobial activity for oral microorganisms", Archives of oral biology, 56, 650-654 (2011).

Yanowitz, J., E. Christensen, and R. McCormick, "Utilization of Renewable Oxygenates as Gasoline Blending Components", (2011).

Noureddini, H., and M.L. Rempe; "Pelargonic acid in enhanced oil recovery", Journal of the American Oil Chemists' Society, 73, 939-941 (1996).

Turnwald, S.E., M.A. Lorier, L.J. Wright, and M.R. Mucalo, "Oleic Acid Oxidation Using Hydrogen Peroxide in Conjunction with Transition Metal Catalysis", Journal of Materials Science Letters, 17, 1305-1307 (1998).
Spargo, P.; "Industrial Organic Chemistry", Completely Revised 4th ed By K. Weissermel and H.-J. Arpe. WileyVCH: Weinheim, (2003).

Godard, A., P. de Caro, E. Vedrenne, Z. Mouloungui, and S. Thiebaud-Roux; "From crops to products for crops: Preserving the ecosystem through the use of bio-based molecules", Oilseeds \& fats Crops and Lipids, 23, (2016). Enferadi Kerenkan, A., F. Béland, and T.-O. Do; "Chemically catalyzed oxidative cleavage of unsaturated fatty acids and their derivatives into valuable products for industrial applications: a review and perspective", Catalysis Science \& Technology, 6, 971-987 (2016).

Khlebnikova, T.B., Z.P. Pai, L.A. Fedoseeva, and Y.V. Mattsat; "Catalytic oxidation of fatty acids. II. Epoxidation and oxidative cleavage of unsaturated fatty acid esters containing additional functional groups", Reaction Kinetics Catalysis Letters, 98, 9-17 (2009).

Enferadi Kerenkan, A., A. Serge Ello, B. Echchahed, and T.-O. Do; "Synthesis of Mesoporous Tungsten Oxide $/ \gamma$ Alumina and Surfactant-Capped Tungsten Oxide Nanoparticles and Their Catalytic Activities in Oxidative Cleavage of Oleic Acid", International Journal of Chemical Reactor Engineering, 14, (2016).

Ichihara, K.I. ,and Y. Fukubayashi, "Preparation of fatty acid methyl esters for gas-liquid chromatography", Journal of lipid research, 51, 635-640 (2010). 